\section{Informations}

Orthop Traumatol (1991) $1: 206$

\section{Juin 1992}

\section{CEPUR Vittel}

Imagerie Clinique du Rachis

Paris, 10, 11 et 12 juin 1992

\section{organisé par M.G. Dupuis et Ph. Peetrons}

sous la responsabilité scientifique de J.L. Dietemann sous la présidence de L. Jeanmart et $\mathrm{A}$. Wackenheim

Informations et inscription : Dr. M.G. Dupuis, Service de Radiologie, Centre de Traumatologie et d'Orthopédie, 10, avenue Baumann, F67403 Illkirch Cedex, Tél. 886733 33, Fax 88674515

\title{
Septembre 1992
}

\section{International post-graduate course}

Bone circulation in normal and pathological conditions

Brussels, Belgium, September 25 and 26, 1992

organized by ARCO (Association Research Circulation Osseous)

Informations : $\operatorname{Pr}$. A. Schoutens, Service de Médecine Nucléaire, Hôpital Universitaire Erasme, 808 route de Lennik, B-1070 Brussels, Belgium, Tel. 3225553 300, Fax 3225554405 